\title{
Massive haemoptysis complicating exogenous lipid pneumonia
}

\author{
M. Haro*, I. Murcia*, A. Núñez*, E. Julia**, J. Valer+
}

Massive haemoptysis complicating exogenous lipid pneumonia. M. Haro, I. Murcia, A. Núñez, E. Julia, J. Valer. OERS Journals Ltd 1998.

ABSTRACT: Exogenous lipid pneumonia is an uncommon condition resulting from the aspiration or inhalation of fat or oil material. The prognosis in this entity is usually good and complications are rare. We report an unusual case of chronic lipid pneumonia complicated with massive haemoptysis who responded to steroid therapy after arterial embolization.

Eur Respir J 1998; 11: 507-508.

\author{
*Respiratory, **Radiology and +Pathol- \\ ogy Services, Hospital General, Albacete, \\ Spain. \\ Correspondence: M. Haro, Sección de Neu- \\ mología (Planta 2), Hospital General, c/ \\ Hermanos Falcó s/n, Albacete, Spain \\ Fax: 34967597121 \\ Keywords: Arterial embolization, lipid pneu- \\ monia, massive haemoptysis \\ Received: June 51997 \\ Accepted after revision August 301997
}

Exogenous lipid pneumonia is an uncommon condition resulting from the aspiration or inhalation of mineral, animal or vegetable oils into the lungs over an extensive period of time $[1,2]$. The prognosis of this entity is usually good if the disorder is correctly identified and the offending agent removed, unless the pneumonitis is so widespread and severe that respiratory failure is caused, or is associated with a superimposed infection. Other complications are rare and include hypercalcaemia due to lipid granulomas, mycobacterial infection, progressive fibrosis or lung cancer [1-4]. We describe a patient with lipid pneumonia complicated with massive haemoptysis who responded to steroid therapy after arterial embolization.

\section{Case report}

A 71 yr old Caucasian man was referred to our institution with massive haemoptysis. He had a $45 \mathrm{yr}$ history of cigarette smoking, but had stopped 9 yrs previous, when he was diagnosed as having a squamous cell carcinoma of the larynx. Laryngectomy with tracheotomy was performed. He had taken liquid paraffin oil as a tracheal lubricant for the past 6 yrs $(3-5 \mathrm{~mL}$ daily). The patient had been well until he presented himself with a 2 day history of cough with blood-tinged sputum, followed by marked haemoptysis (approximately $600 \mathrm{~mL}$ in $24 \mathrm{~h}$ ). On physical examination, the right basal region of the lung was dull on percussion. Breath sounds were diminished but cardiac examination was normal. There were no peripheral oedemas, clubbing or cyanosis. A chest radiograph showed a homogeneous round right lower lobe consolidation. The erythrocyte sedimentation rate was $48 \mathrm{~mm} \cdot \mathrm{h}^{-1}$, haemoglobin was at a concentration of $15 \mathrm{mmol} \cdot \mathrm{L}^{-1}$, haematocrit $44 \%$ and the white blood cell count was $8.7 \times 10^{9}$ cells $\cdot \mathrm{L}^{-1}$ with neutrophils, lymphocytes and monocytes present at 78,14 and $8 \%$, respectively. Rheumatoid factor, complement levels, antineutrophil cytoplasmatic autoantibodies (ANCA), carcinoembryogenic antigen (CEA) and serum angiotensin-converting enzyme (SACE) were normal. Arterial blood gas analysis (breathing room air) showed: $\mathrm{pH}$ 7.4; arterial oxygen tension $\left(\mathrm{Pa}, \mathrm{O}_{2}\right) 8 \mathrm{kPa}(60 \mathrm{mmHg})$; carbon dioxide tension $\left(\mathrm{Pa}_{\mathrm{a}} \mathrm{CO}_{2}\right) 5.3 \mathrm{kPa}(40 \mathrm{mmHg})$; and arterial oxygen saturation $\left(\mathrm{Sa}_{\mathrm{a}} \mathrm{O}_{2}\right)$ of $90 \%$. An electrocardiogram was normal, a tuberculin skin test was negative and three sputum studies disclosed no acid-fast bacilli (AFB). Bronchoscopic examination showed the trachea and lower airways diffusely stained with bright red blood, with active bleeding from the right lower lobe. Bronchial arteriography and selective catheterization of a dilated bronchial artery in the region of the right lower lobe was performed. Multiple pellets of microencapsulated absorbable gelatin sponge (Gelfoam) were injected, resulting in the occlusion of the bleeding artery and complete disappearance of haemorrhage. A computed tomographic scan

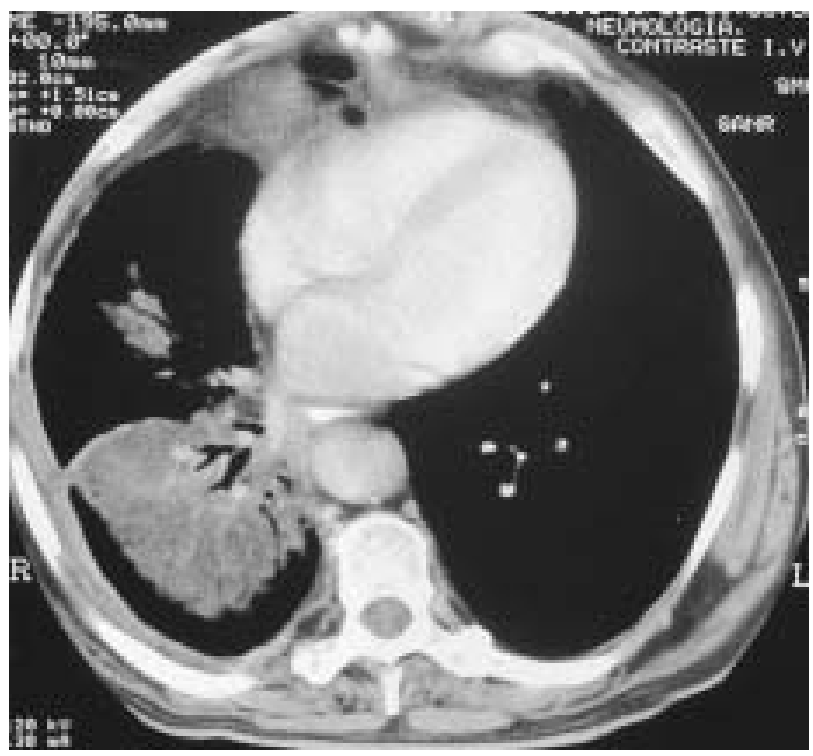

Fig. 1. - Computed tomography scan of the chest. Right lower lobe consolidation with air bronchograms and pleural thickening. 


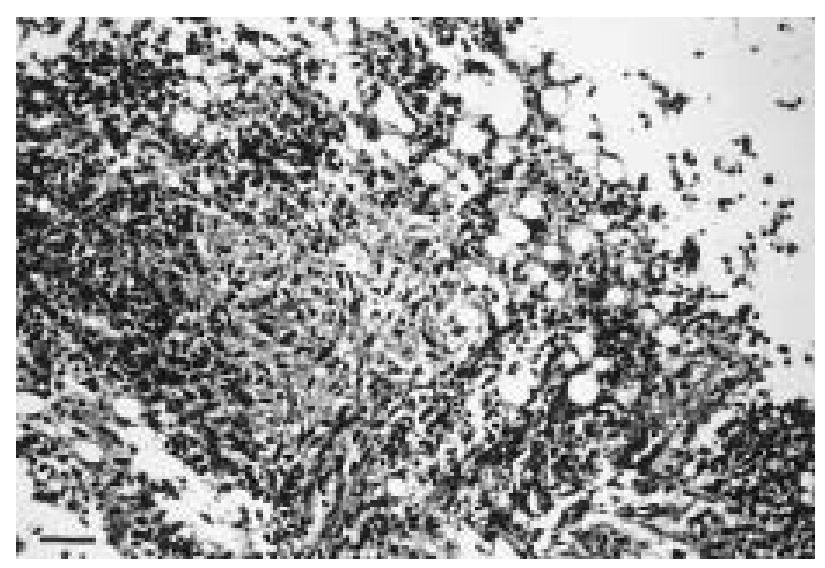

Fig. 2. - Transbronchial biopsy. Intense inflammatory reaction with a poorly-delineated noncaseating granuloma surrounded by empty vacuoles corresponding to lipids, free and within macrophages. (Haematoxylin and eosin stain, internal scale bar $=50 \mu \mathrm{m}$ ).

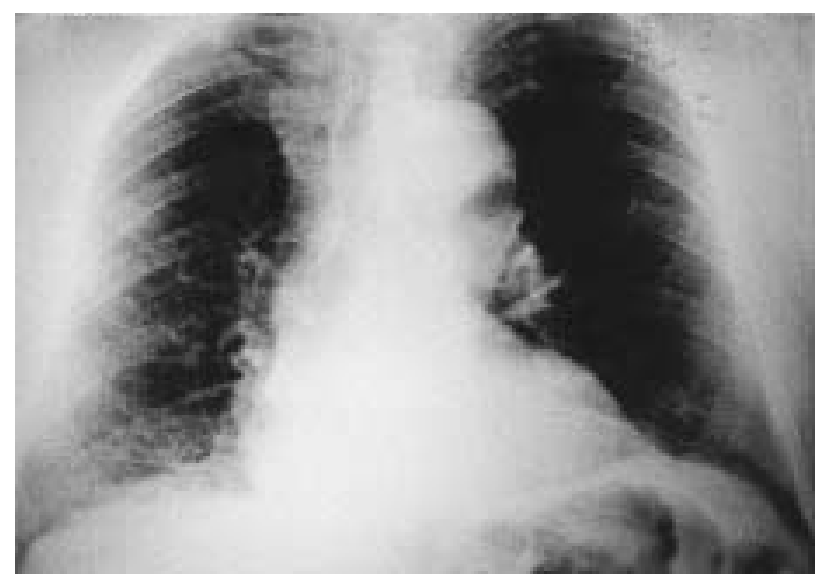

Fig. 3. - Chest radiograph. Almost complete resolution of right lower lobe consolidation after corticosteroid therapy.

of the thorax revealed two different right lower lobe consolidations with air bronchograms and fat within pulmonary tissue (values of attenuation - 132 hounsfield units (HU)), pleural thickening, but no enlarged lymph nodes (fig. 1). On the fourth hospital day, bronchoscopy showed an inflamed bronchial mucosa in the right lower lobe but no endobronchial lesion could be seen. Transbronchial biopsies were performed. Microscopically, the biopsies showed multiple poorly-delineated noncaseating interstitial granulomas surrounded by an intense inflammatory reaction and lipid-ladden macrophages (positive for the stain Sudan IV), similar to a foreign body cell reaction (fig. 2). Microbiological and cytological examinations of the bronchial washings and biopsies revealed no fungi, AFB or atypical cells. The patient was diagnosed as having an exogenous lipid pneumonia, and prednisolone was administered as a single oral dose for 6 weeks (40 mg daily), which was gradually tapered to zero and stopped after 3 months. A chest radiograph showed marked improvement after the first month and almost complete resolution after 6 months (fig. 3).

\section{Discussion}

The clinical course of haemoptysis is unpredictable and a small sentinel bleeding may herald massive, potentially fatal haemorrhage. More than 40 diseases cause massive haemoptysis and approximately $90 \%$ of the reported cases have been due to benign diseases (chronic cavitary tuberculosis and inflammatory lung disease) [5]. About half of the patients with lipid pneumonia have no symptoms and haemoptysis is rare [3]. To our knowledge, this is the first reported case of lipid pneumonia presenting with massive haemoptysis. We assume that the chronic use of paraffin oil resulting in a vigorous inflammatory response and foreign body reaction in the lung with new vessel formation, extensive remodelling and hypertrophy of the bronchial artery. Bleeding was probably caused by repeated infection and progressive pulmonary involvement.

The therapeutic approach most frequently adopted in lipid pneumonia is discontinuation of the oil exposure [6]. Surgery is seldom required and corticosteroids or therapeutical bronchoalveolar lavage have not been demonstrated to be effective in all cases $[3,7,8]$.

Our case illustrates the diagnostic and therapeutic role of arterial embolization, as opposed to surgery, in the management of massive haemoptysis in benign localized disease. Because of the severity of the inflammatory cell reaction and based upon previously reported cases in which lipid pneumonia was successfully treated, a trial of corticosteroid therapy was started. The prompt response, with almost complete radiographic resolution, made thoracotomy unnecessary, and confirmed that corticosteroids could be used in selected patients [7]. However, it is very important to exclude a nontuberculous mycobacterial infection before starting this therapy because of the known association with lipid pneumonia [1].

\section{References}

1. Spickard A, Hirschmann JV. Exogenous lipid pneumonia. Arch Intern Med 1994; 154: 686-692.

2. Wright BA, Jeffrey PH. Lipid pneumonia. Semin Respir Infect 1990; 5: 314-321.

3. Gondouin A, Manzoni Ph, Ranfaing E, et al. Exogenous lipid pneumonia: a retrospective multicentre study of 44 cases in France. Eur Respir J 1996; 9: 1463-1469.

4. Lores L, Ribas J, Ruiz J, Cuxart A, Frignola A, Morera J. Acute lipoid pneumonia. Presentation of two cases. Med Clín (Barc) 1996; 106: 747-748.

5. Cahill BC, Ingbar DH. Massive hemoptysis. Assessment and management. Clin Chest Med 1994; 15: 147-168.

6. Romero S, Martín C, Palau M, Tobías I, Aranda I, Escortell E. Lipoid aspiration pneumonia. Arch Bronconeumol 1989; 25: 28-30.

7. Nyat-Kooi Chin M, Kok-Pheng Hui, Rajalingam Sinniah, Tiong-Beng Chan. Idiopathic lipoid pneumonia in an adult treated with prednisolone. Chest 1994; 105: 956-957.

8. Casademont J, Xaubet A, López J, Agustí C, Ramírez J. Radiographic bilateral cavitary lesions in lipoid pneumonia. Eur Respir J 1988; 1: 93-94. 